\title{
Holographic Recording through Tiny Chamber's Vacuum as a Microscopic Installation Art
}

\author{
Diaa Ahmed Mohamed Ahmedien \\ Drawing and Painting Dept., Faculty of Art Education, Helwan University, Cairo, Egypt
}

\begin{abstract}
In this artistic and scientific experiment I try to build essential $3 D$ common links with our communities by one of the most important scientific approaches in laser arts; it is holographic communication in contemporary visual arts, I seek to invoke extraordinary methods to design and record spatial field of microscopic installation art, which allow to unleash the theoretical and practical information about new rare kinds of visual arts in wide ranges of our communities, which could be able to make them pass through paradigm shift in their visual experiences and conceptual towards more interaction with contemporary visual arts trends, as an attempt to emphasize to the mature relationship between the art, science and technology through the latest scientific and artistic mutations around the world.
\end{abstract}

Keyword: Holography, Laser Art, Microscopic Art, Installation Art

\section{Introduction}

The term hologram has been used -often incorrectly- to mean many things - encompassing everything from a projected 3D image floating in the air, to lenticular posters, to sparkly Christmas wrapping paper. This research tries to define a hologram accurately and visually as a record of the interaction of two mutually coherent light beams (Laser) in form of microscopic pattern of interference fringes. And ask to what extent holography can explain how our brains can store so many common memories in so little space? Interestingly, how also possess a fantastic capacity for information storage? By changing the angle at which the two lasers strike a piece of photographic film, it is possible to record many different images on the same surface. Any image thus recorded can be retrieved simply by illuminating the film with a laser beam possessing the same angle as the original two beams. By employing this method, references have calculated that one inch-square of film can store the same amount of information contained in fifty large books. And now this research tries to enhance the role of holographic communication technique as an important part of sciences of visual arts and its role to help artists to integrate with contemporary visual arts trends in different societies to product new forms of expression that allow them to explore a visual perception of the world in a different perspective. The "abstract" option is an open door to develop the creativity that artists aspire to achieve with their work. Also, each viewer has their own perception of what is exposed to them, so each interpretation will be different leading to the evolution of newer and more creative methods of art making.

\section{Material And Methodology}

The material and methodology in this experiment will be consisted into three sections, which related to practical and theoretical information that need to be recorded holographically. First the panchromatic holographic system must be tested; second i collected a lot of kinds of liquid materials that fit to be mixed with each other and the third is holographic record process for spatial objects. This experiment conducted by using three kinds of holographic setups: Single Beam setup, Double Beam -Reflection- setup and Double Beam Transmission- setup. Figure 1 shows the differences between both of them. 


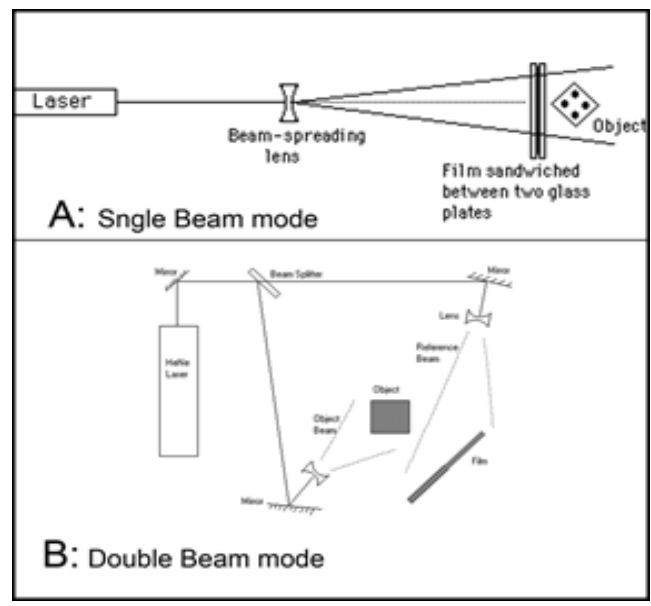

Fig: 1 shows the different holographic setup in both of single and double mode

Finally the essential material that must be existed (Holographic plates or film, Developing chemicals, Laser, Beam spreader, Beam splitter and Optical table).

Since Russian panchromatic plates came on the market twenty years ago, progress has been made in true color holograms (Hubel, 1991; Bjelkhagen \& Vukicevic, 1991; Bjelkhagen etal., 1996). The various chemical treatments applied to these plates are explained in detail by several authors (Bjelkhagen, 1993; Sasomov, 1999). The plates used are silver-coated single film PFG 03C plates from the Slavich Company in Moscow. Their chemical treatment first includes a hardening of the gelatin, development, bleaching, a series of rinses in alcohol and slow drying. The hologram's spectral characteristics were analyzed by taking a double exposure holographic interferogram and placing a small mirror near the object to be analyzed, in order to make a spectral analysis of the reconstructed light waves. The spectrograms of the reconstructed waves in our very first validation tests showed that the three peaks corresponding to the reconstructed colors are slightly shifted by a few nanometers, which corresponds to the contraction of the gelatin thickness:

. $471 \mathrm{~nm}$ for the blue, or ...-5 nm,

$.511 \mathrm{~nm}$ for the green, or ....- $3 \mathrm{~nm}$,

$.640 \mathrm{~nm}$ for the red, or .... $-7 \mathrm{~nm}$

These differences are reduced practically to zero in wind tunnel tests when the hologram is placed normal to the bisector of the angle formed by the object and reference beams.

Fig. 2 shows the feasibility setup implemented in the laboratory. The Innova Spectrum laser emits eleven lines in the visible simultaneously. The red, green, and blue lines we want are diffracted by an acoustooptic cell in which are generated three frequencies $\mathrm{f} 1, \mathrm{f} 2$ and $\mathrm{f} 3$ appropriate to the three wavelengths $.1, .2$ and .3. A beam splitter cube splits the reference beams and three object beams. The three reference beams are collimated onto the holographic plate by an achromatic lens located a focal length from the pinhole (diameter 25 .m) of a spatial filter having a microscope objective lens (x60). The three object beams are collimated the same way to form three parallel light beams between two large achromatic lenses and illuminate the test section.

The hologram is thus illuminated on the same side by the three parallel reference beams and the three convergent measurement waves. A diaphragm is placed in the focal plane just in front of the camera in order to be able to filter out any parasitic interference. The hologram is first illuminated in the absence of flow and is then developed and placed back in exactly its original position. When the hologram is illuminated with the reference beam, nine diffraction images are seen. 


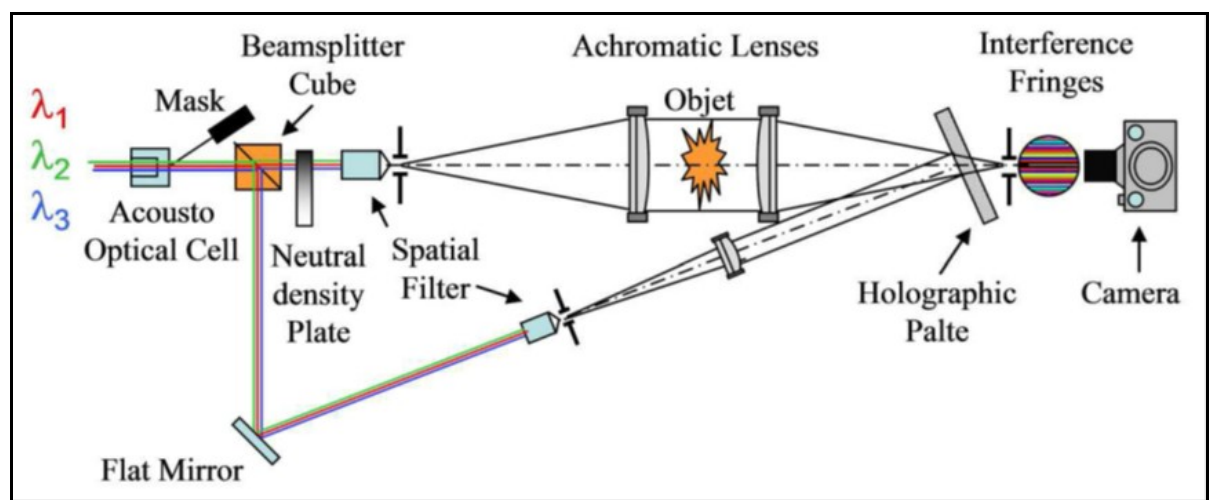

Fig. 2. Real-time color holographic interferometry setup (Transmission mode)

\section{Experimental Setup}

In the Laser Institute at Cairo University, the experiment has been conducted within 50 days at a rate of ten individual objects a day. I used single beam mode for $2 \mathrm{~d}$ object and double beams for $3 \mathrm{D}$ objects. Figure one shows the different holographic setup in both of single and double mode. Ten people were existed with me every day to record their objects on slides that were about $10 \mathrm{c} . \mathrm{m} \times 10 \mathrm{c} . \mathrm{m}$.

The main technical concept in this experiment is relationship between references beam (RB) and object beam $(\mathrm{OB})$, to better understand this experiment; we start with introducing a $\mathrm{Q}$ factor that defines two different kinds of holographic gratings-thin (plane) grating and thick (volume) grating. In a thin grating (periodic lines structure), each line acts as an individual scattering center and there is no talking" between them. The far field diffraction pattern is formed by the superposition of each scattering wavelet. While in a thick grating (periodic planes structure), the incident wave and diffracted wave are coupled together as they propagate along the thick periodic structure and there's energy transfer between them. In this case, strong diffraction can only be seen when the Bragg condition is met. The $\mathrm{Q}$ factor is defined as:

$$
Q=2 \pi \frac{\lambda / n}{\Lambda} d / \Lambda
$$

In which $K$ is the incident light wavelength in vacuum, $\mathrm{n}$ is the refractive index of the emulsion, $\Lambda$ is the grating period, and $d$ is the emulsion thickness.

\section{Optical setup around the chamber:}

The optical setup is shown in Fig. 3. The beam power as it leaves the laser is $1.80 \mathrm{~W}$ when the etalon is set perpendicular to the laser beam axis and $1.20 \mathrm{~W}$ when it is tilted. The polarization of the three beams rotates $90^{\circ}$ at the exit from the acousto-optic modulator, so that the polarization vectors lie parallel to the reflecting surfaces of the mirrors. This arrangement makes it possible to have beams of the same polarization interfere on the hologram. The three wavelengths downstream of the acousto-optic cell are split into a reference beam and object beam by a beam splitter cube. A right angle prism is used to adjust the reference and object path lengths on the hologram. A spatial filter is used to expand the beam for its passage through the test section. A pair of achromatic lenses converts the beam into parallel light in the test section and then focuses it on the hologram.

The reference beam passes over the test section, and then another achromatic lens is used to illuminate the hologram with a parallel light beam. For reference, the object beam diameter is $40 \mathrm{~mm}$ at the hologram and that of the reference beam is $60 \mathrm{~mm}$. At the acousto-optic cell, the power of the three light waves is practically the same (of the order of $70 \mathrm{~mW}$ per channel). The beam splitter cube distributes $85 \%$ of this power to the reference path and $15 \%$ to the measurement path. 


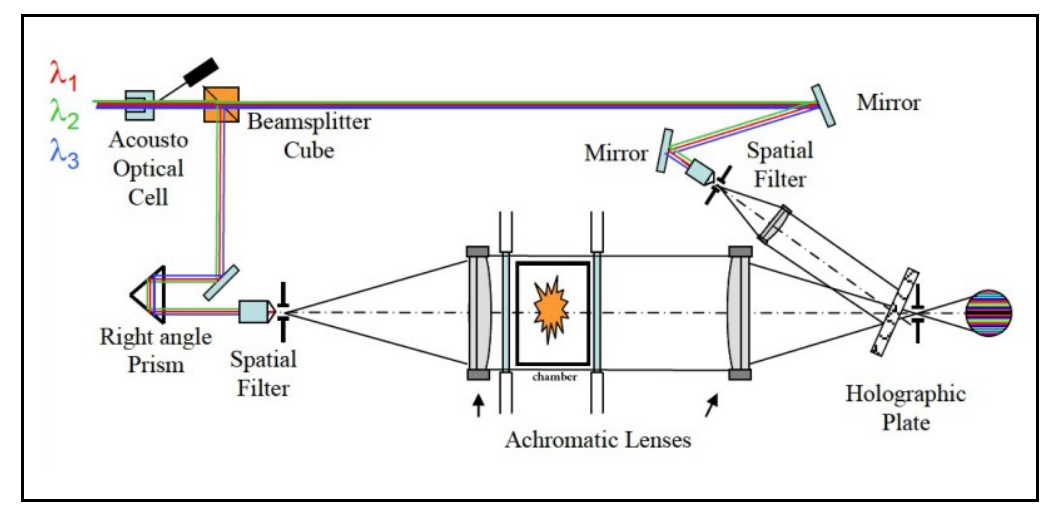

Fig. 3. Optical setup implemented around the chamber

Developing the film is the second critical step, and a myriad of developing techniques and recipes exist. The following one is that of T.H. Jeong from Lake Forest College. The Part A solution, Part B solution and Bleach solution are prepared from the JD-2 chemical processing kit and they are prepared for you before the lab. The following procedures will be carried on in separated dark rooms as follows:

1. Develop: Quickly submerge the plate into the developer so that all parts get wet evenly. Slush it around for about two minutes. The hologram should turn almost black.

2. Rinse: Rinse the developed hologram with agitation for at least 20 seconds. For best results and longer lasting holograms, rinse up to three minutes to make sure that all of Part A has been rinsed.

3. Bleach: Place the rinsed hologram into the bleaching solution; agitate it until the plate is completely clear (this may take up to two minutes); then, bleach for another 10 seconds.

4. Rinse again: Rinse the bleached hologram with agitation for at least 20 seconds (up to 3 minutes).

5. Dry: Stand the plate vertically with a paper towel under it, dry in room temperature.

To collect these slides after developing processes in one board, I designed glass covers for every one of them. In the Setup for reconstructing the image we use the same reference beam as in the expose setup. The reference beam is directed by the recorded fringe patterns on the plate in a way such that the directed beam seems to come from where exactly the original object locates.

\section{Result And Discussion}

Theoretically, the entire size of slides was almost 50 meters $\times 50$ meters $(500$ slides every one of them $10 \mathrm{~cm} \times 10 \mathrm{~cm}$ ) of holographic memories for all objects, then decode these symbols by holographic reconstruction image in the installation art exhibition, therefore this entire capacity space will carry almost billion of nontouchable and visible holographic memories simultaneously by this artistic experiment through this kind of scientific process arts.

In terms of real objects, figure 4 shows the spatial light field that has been recorded by double beam setup. It is clear that the image can be reconstructed accurately with real dimensions. Figure 5 also shows the variety of vision that resulted of change the angle of recording, after all from this experiment we can find that:

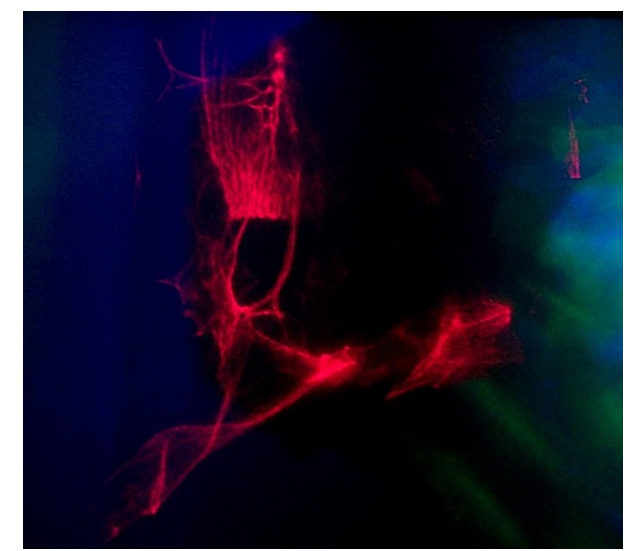

Fig. 4 shows the spatial light field that has been recorded

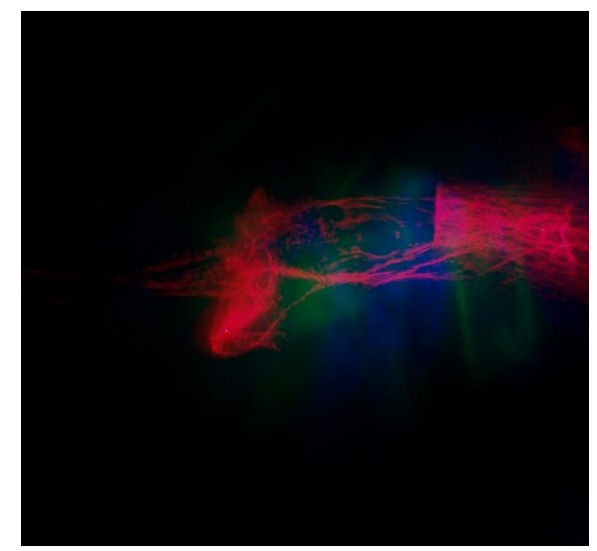

Fig. 5 variety of vision that by changing the angle of recording 
1- Holographic techniques have a lot of abilities to record visual data by different modes.

2- Holographic techniques fit to represent a lot of visual memories and discover unknown aspects in them.

3- Interactive art processes can be enriched by using the holographic techniques.

4- People were so active with the new scientific techniques in experimental visual arts.

5- The relationship between art, science and technology must be translated into practical artistic activities with wide ranges of our communities.

6- Experimental visual artistic values in the interactive process depend on new scientific techniques.

\section{Conclusion}

This experiment tries to discover the common relation among our accumulated memories in the past and our future as an extension of them. By relying on the theory of information in the contemporary sciences of visual arts, we can deal of ourselves as visual information that put in visual environment also, and here the holographic techniques can translate ourselves into visible wavelengths (coding process),. Therefore in the gallery the information in wavelengths will be reconstructed to return into original visual message (decoding process). So, this scientific process can make us able to see ourselves as an extension in the past towards common determination, and try to answer: what our memories made of? Where the role of driving force to our memories to change our relationships, cultures and identities?

\section{Acknowledgements}

First and foremost I cannot deny the role of British council in this experiment, where it has been granted totally by the British Council fund system for artists. Second this experiment has been conducted in the Institute of Laser Sciences at Cairo University, so I would like to thank both of British Council and Laser institute for their great contributions for my experiment.

\section{References}

[1]. Aren Ben- Dor, "Law and Art -Justice, Ethics and Aesthetics-", Taylor \& Francis group, Rutledge, First edition, London, UK, 2013.

[2]. Charlie Gere, "Digital Culture", REAKTION Book, Second edition, London, UK, 2008.

[3]. Charlotte Peter Fiell, "Design Now", TASCHEN, First edition, London, UK, 2010

[4]. Edward Shanken, "Art and Electronic Media", PHAIDON, First edition, New York, USA, 2009.

[5]. Francis Franciscan, "Modern Art Culture", Taylor \& Francis group, Rutledge, Second edition, London, UK, 2009.

[6]. Jack Ferrier, "Visual Documents", European Communities - Research group-, extended edition, Brussels, Belgium, 2009.

[7]. James Elkins, "Theorizing Visual studies - write through the discipline-", Taylor \& Francis group, Rutledge, First edition, London, UK, 2013.

[8]. Janze Polojonik, "Art and Science", European Communities - Research group, Luxembourg, 2008.

[9]. Marshal McLuhan, "Understanding Media", Taylor \& Francis group, Rutledge, third edition, London, UK, 2013.

[10]. Micho Kaku, "Physics of the Impossible", BOUBLEDAY, First edition, New York, USA, 2008.

[11]. Oliver Herwing \& Axel Thallemer, "Unity of Art and Science", ARNOLD SCHEN, First edition, New York, USA, 2011.

[12]. Paul Zelanski \& Marry Pat fisher, "Shaping and Space - the dynamics of three dimensions designs-", THOMSON WADSOURTH, First edition, New York, USA, 2007.

[13]. Roy Ascotte, "Art, Technology and consciousness", INTELLECT, Bristol, UK, 2005.

[14]. Stephen Wilson, "Art and Science Now", THOMSON WADSOURTH, First edition, San Francisco, USA, 2010.

[15]. Alicia. M. Gibb, "New Media Arts in Micro Controller Technology", -Analytical study-, Ph.D. thesis, -published- School of Art and Design, University of Manchester, UK, 2006.

[16]. Alf. L. Nay, "Long term of Art and Science of Collaboration", Philosophical study-, Ph.D. thesis, -published- Faculty of Visual Arts, University of London Gold themes, London, UK, 2007.

[17]. George Lugosi, "Symmetry, Art and Science: Philosophical approach", Ph.D. thesis, -published- School of Media Arts, Victoria University, Victoria, Australia, 2009.

[18]. Gil. Dekel, "Scientific Inspiration: approach to create contemporary Media art works", Ph.D. -published- thesis, Visual Arts College, University of Colorado, Colorado, USA, 2006

[19]. Heida. A. Rngd Atter, "Media Art Theories - Analytical Studies", Ph.D. thesis, -published- School of Visual arts, University of British Colombia, Colombia, Canada, 2010.

[20]. Jasmia. Kara beg, "Micro Science Progress through Visual art trends -Analytical studies", Ph.D. thesis, -published- School of Visual arts, University of British Colombia, Colombia, Canada, 2009.

[21]. Silvana. Barbosa, "From Fine Art to Natural Science through allegory", Ph.D. thesis, -published- School of Art and Science, University of New Castel, New Castel, UK, 2008.

[22]. Edward. Shanken, "The Technology Role in Redefine Conceptual art -Analytical study-", Ph.D. thesis, -published- Faculty of Visual Arts, University of London Gold themes, London, UK, 2010.

[23]. Anders Ramirez, "When is Information Visualization art", Journal of Visual Language and Computing, ELSAVIER, Vol:453/12, Jun, 2007.

[24]. Bell Wester, " Logarithm of visual arts", published research, center of visual arts and technology, University of Michigan, New york, USA, 2011.

[25]. Diaa Ahmed Mohamed Ahmedien, " The modern use of optics design techniques to product visual Art works", IOSR Journal of Humanities and Social Science, ESAVIER, Vol: X, Issue: X, Sep-Oct. 2012. 